\title{
Rapid identification of gram-negative bacilli from blood cultures
}

\author{
M. STEVENS and G. T. PARISH
}

\section{Public Health Laboratory, Royal Infirmary, Leicester, LE1 5WW}

\begin{abstract}
Summary. Blood-culture broths showing macroscopic or radiometric evidence of growth of gram-negative bacilli were examined by a rapid automated bacterial identification system. A differential centrifugation technique was developed to prepare suitable inocula. The identification results obtained were confirmed by the API $20 \mathrm{E}$ method, with single colonies of the strains isolated 24 h later. Of 90 organisms tested, seven did not give the same identification by the two systems. With the rapid automated technique a presumptive identification of gram-negative bacilli can be made $24 \mathrm{~h}$ earlier than by more conventional methods.
\end{abstract}

\section{Introduction}

Several methods for the rapid detection and identification of organisms directly from blood cultures have been described. Some require overnight incubation of the identification system, for instance those of Blazevic et al. (1976) and Furtado and Medeiros (1982), whereas some methods provide results after incubation periods of only $4-5 \mathrm{~h}$, for instance those of Wasilauskas and Ellner (1971), Malloy et al. (1983) and Mounier and Denis (1983). Useful information including presumptive antimicrobial susceptibility can be available on the same day that the blood culture has been found to be positive.

In the study described here we used the Quantum II bacterial identification (BID) system (Abbott Laboratories, Wokingham, Berkshire) in which tests are incubated for 4-5 h and the interpretation of test results and calculations of identification probabilities are automatic (Murray et al., 1984; Stevens et al., 1986). The blood-culture specimens examined were of three types: conventional homeprepared bottles of media, a radiometric method and a commercially available system. A differential sedimentation technique was developed to provide a pellet of organisms from bottles showing evidence of growth. The pellet was used to prepare an inoculum for the Quantum II BID cartridge. The identifications of the organisms obtained by the Quantum II BID system were confirmed by the API 20E method.

Received: 5 Mar. 1985; accepted: 21 May 1985.

\section{Materials and methods}

\section{Blood-culture systems}

A total of 90 blood cultures of three types was examined. All included an aerobic and an anaerobic bottle.

Conventional media comprised an aerobic bottle containing tryptic soy broth plus sodium polyanethol sulphonate (Liquoid) $0.025 \%$ and para-amino-benzoic acid $0.005 \%$ and an anaerobic bottle containing thioglycollate broth plus para-amino-benzoic acid $0.005 \%$ and agar $0.1 \%$.

The Bactec model 460 radiometric system (Laboratory Impex, Twickenham, Middlesex) was used with 6B aerobic and 7D anaerobic media. These were tryptic soy broth plus additives and contained ${ }^{14} \mathrm{C}$-labelled substrates.

The Septi-Chek system (Roche Products, Welwyn Garden City, Herts) comprised a pair of aerobic and anaerobic bottles. The former had a dip slide attached with chocolate agar, MacConkey agar and malt agar surfaces.

A series of 67 conventional sets, 12 Bactec sets and 11 Septi-Chek sets was examined. The commercial systems were used according to the manufacturers' instructions: 5 $\mathrm{ml}$ of blood was added to all types of bottle. Contents of bottles found to be radiometrically or visually (turbid) positive, were initially examined microscopically with Gram's stain. Only cultures containing morphologically similar gram-negative rods were included in this study.

\section{Differential centrifugation}

A differential centrifugation technique was performed to provide a suitable inoculum for the identification system. Approximately $10 \mathrm{ml}$ of mixed contents of the blood-culture bottle was decanted into a $20-\mathrm{ml}$ "V bottomed" screw-capped container. Sterile distilled water was added to make a total of $20 \mathrm{ml}$, thoroughly mixed 
with a vortex mixer, and the mixture centrifuged at $200 \mathrm{~g}$ for $5 \mathrm{~min}$. The supernate was removed and retained, the deposit being discarded. Sterile distilled water was added to make a total of $20 \mathrm{ml}$ and the mixture was centrifuged at $1650 \mathrm{~g}$ for $5 \mathrm{~min}$. The supernate was removed and $20 \mathrm{ml}$ of sterile distilled water was added to the pellet. This second centrifugation step was repeated twice. A cytochrome oxidase test (Kovacs, 1956) was then performed on a portion of the bacterial pellet. The remainder of the pellet was resuspended in sterile distilled water to achieve an opacity matching a McFarland 0.5 barium sulphate standard.

\section{Bacterial identification}

The suspension was used to inoculate a Quantum II BID cartridge as recommended by the manufacturer. Cartridges were incubated at $37^{\circ} \mathrm{C}$ for $4 \mathrm{~h}$, after which an indole test was performed on chamber 18 of the cartridge, and that result, together with the result of the oxidase test previously performed, was entered into the Quantum II instrument. The cartridge was then read automatically and an identification of the isolate, with a probability score, produced.

The blood culture specimens were all subcultured on to horse blood agar, heated blood agar and MacConkey agar. After overnight incubation at $37^{\circ} \mathrm{C}$, respectively anaerobically or in an atmosphere of air plus $\mathrm{CO}_{2} 10 \%$ or aerobically, isolates were identified by the API 20E (API, Basingstoke, Hants) method according to the manufacturer's instructions; the inoculum was prepared from a single isolated colony. Comparisons were then made between the results obtained from the two identification methods. When a disagreement occurred appropriate additional conventional tests (Cowan, 1974) were performed to obtain a definitive identification.

\section{Results}

The total number of blood cultures examined was 90. The Quantum II BID result was confirmed by the API 20E system in 83 cases (table I).

The seven strains for which the identities differed are shown in table II. In four instances the API result was vindicated by conventional tests. With two strains ( 1 and 2) the Quantum II BID result was equivocal and with one (strain 3 ) the data base was inadequate. We considered the Quantum II BID identifications to be equivocal when the probability was $<80 \%$. Some API results also suggested that the strains were atypical by including the comments "Low selectivity" or "Acceptable" after the identification, rather than "Excellent" or "Very Good Identification". Strain 2 was a typical Escherichia coli, but the glucose test result was negative by the Quantum II BID system and this was solely responsible for the discrepancy. Strain 3 was an atypical $E$. coli which was recorded as citrate positive by the Quantum II BID system only. Incubation for $48 \mathrm{~h}$
Table I. Strains isolated for which the Quantum II BID results were confirmed by API $20 \mathrm{E}$

\begin{tabular}{lc}
\hline Species & Number \\
\hline Escherichia coli & 38 \\
Enterobacter cloacae & 7 \\
Klebsiella pneumoniae & 6 \\
Klebsiella oxytoca & 4 \\
Citrobacter freundi & 1 \\
Proteus mirabilis & 3 \\
Proteus vulgaris & 3 \\
Citrobacter diversus & 2 \\
Enterobacter aerogenes & 1 \\
Morganella morgani & 1 \\
Salmonella enteritidis & 1 \\
Pseudomonas aeruginosa & 8 \\
Acinetobacter lwoffi & 2 \\
Pseudomonas maltophilia & 2 \\
Acinetobacter calcoaceticus & 2 \\
Alcaligenes sp. & 1 \\
Not in data base* & 1 \\
Total & 83 \\
\hline
\end{tabular}

* Haemophilus influenzae.

Table II. Discrepancies between Quantum II BID, API $20 \mathrm{E}$ and conventional identification results

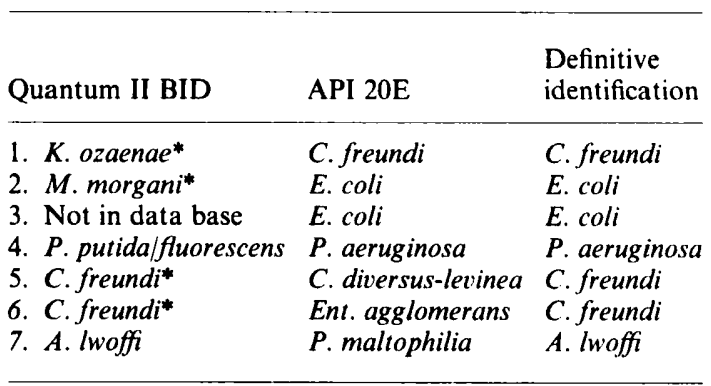

* Equivocal results; additional tests required to confirm identification.

was required to give positive ONPG, lysine and citrate results by the API method for strain 7. An excellent identification of Pseudomonas maltophilia was then obtained. These tests gave negative results in the Quantum II BID system which gave an identification of Acinetobacter lwoffi. We consider that the three positive API test reactions were falsely positive due to the prolonged incubation. These reactions were negative by conventional methods. The acetamide test was falsely recorded as negative by the Quantum II BID system for strain 4. This was due to the effect of the acidic indole reagent in the adjacent compartment. The resultant 
identification was $P$. putida/fluorescens rather than $P$. aeruginosa. The carry-over of indole reagent has been recognised by the manufacturer who now recommends a non-acidic indole reagent.

\section{Discussion}

The study demonstrated that presumptive identifications with a high confidence level could be available on the same day that a blood culture was detected as positive. The information obtained would be useful in selected clinical situations.

The combination of Quantum II BID identification and the Bactec blood-culture method was the optimal system for providing rapid results. On two occasions bottles were positive radiometrically when there was no macroscopic evidence of growth. Our results were similar to those obtained in other studies in which various identification systems were used to examine organisms directly from blood cultures. A parallel direct comparison of the identification results obtained by the two systems (Quantum II BID and API 20E) was not appropriate in this study as the source of the bacterial inoculum for each identification system was fundamentally different. The standardised, recommended method in which single isolated colonies are used was only possible with the API system. When the seven strains for which the results of the two methods disagreed were tested by conventional methods, the Quantum II BID system gave four misidentifications and the API method three.

We considered that the citrate test was most likely to be falsely recorded as positive due to blood residue in the inoculum, as reported in a previous study (Mounier and Denis, 1983). This occurred on one occasion in our study. We advise that the procedure described above be strictly adhered to. If a false positive citrate test was suspected, use of the EDIT mode of the Quantum II BID system, which allowed results of individual tests to be changed, was employed.

Although we mixed each blood culture before the initial centrifugation, to mimic the most adverse

\section{REFERENCES}

Blazevic, D J, Trombley C M, Lund M E 1976 Inoculation of API 20E from positive blood cultures. Journal of Clinical Microbiology 4:522-523.

Cowan S T 1974 Cowan and Steel's Manual for the Identification of Medical Bacteria, 2nd edn. Cambridge University Press, Cambridge. conditions, subsequent testing has indicated that this is unnecessary. The supernate of a blood culture contained sufficient numbers of bacteria for an inoculum, and the efficacy of the centrifugation technique was improved with fewer erythrocytes initially present. The relatively slow initial centrifugation removed most of the blood components. Any remaining erythrocytes were lysed by the distilled water and removed during subsequent washing. There were always enough organisms present to obtain a concentration equivalent to a McFarland 0.5 standard. In many cases there was a considerable excess. Although the data base of the Quantum II BID system included a limited number of taxa which are non-fermenters, we considered this a useful facility. It was possible to identify 15 of 17 such strains within $5 \mathrm{~h}$.

The preparatory techniques described require only equipment normally found in a modest microbiology laboratory, and the methods require a minimal extension of routine procedures. The costs of the disposable elements in both identification systems are similar; actual costs depend upon quantities used. The Quantum II spectrophotometer is provided without charge for use with the BID and other diagnostic tests.

By correlating the identification of the organism with known patterns of antimicrobial susceptibility, valuable presumptive information would be available to the physician on the same day that a blood culture was detected as positive. The antimicrobial susceptibility results could also be obtained rapidly by use of an inoculum taken directly from the blood culture broth in a rapid automated sensitivitytesting system such as the Autobac (General Diagnostics, Abingdon, Oxfordshire) or the MS2 (Abbott Laboratories).

From the findings of this study, the method described can be recommended as a reliable procedure for reporting the presumptive identification of gram-negative bacilli from blood cultures in 4-5 h.

We thank Dr R.A. Swann and Mrs M.A. Foster for their helpful advice.
Furtado G L, Medeiros A A 1982 Rapid biochemical identification of Gram-negative bacilli from blood cultures using API 20E strips. American Journal of Clinical Pathology 78:356 357.

Kovacs N 1956 Identification of Pseudomonas pyocyanea by the oxidase reaction. Nature 178:703.

Malloy P J, Ducate M J, Schreckenberger P C 1983 Comparison of four rapid methods for identification of Enterobacteria- 
ceae from blood cultures. Journal of Clinical Microbiology 17:493-499.

Mounier M, Denis F 1983 Four-hour direct identification of Enterobacteriaceae in blood cultures. European Journal of Clinical Microbiology 2:593-595.

Murray P R, Gauthier A, Niles A 1984 Evaluation of the Quantum II and Rapid E identification systems. Journal of Clinical Microbiology 20:509-514.
Stevens M, Henrichsen C, Smith M 1986 A collaborative evaluation of a rapid, semi-automated identification system for gram-negative bacilli: the Quantum II BID. Journal of Medical Microbiology 21:145-150.

Wasilauskas B L, Ellner P D 1971 Presumptive identification of bacteria from blood cultures in four hours. Journal of Infectious Diseases 124:499-504. 